\title{
Calibration Results of the EBIT Medium-Energy Flat- Field Spectrometer using the LBL Advanced Light Source
}

J.K. Lepson, P. Beiersdorfer, G.V. Brown, H. Chen, E.M. Gullikson, M.B. Schneider, S.B. Utter, K.L. Wong

\section{January 17, 2001}




\section{DISCLAIMER}

This document was prepared as an account of work sponsored by an agency of the United States Government. Neither the United States Government nor the University of California nor any of their employees, makes any warranty, express or implied, or assumes any legal liability or responsibility for the accuracy, completeness, or usefulness of any information, apparatus, product, or process disclosed, or represents that its use would not infringe privately owned rights. Reference herein to any specific commercial product, process, or service by trade name, trademark, manufacturer, or otherwise, does not necessarily constitute or imply its endorsement, recommendation, or favoring by the United States Government or the University of California. The views and opinions of authors expressed herein do not necessarily state or reflect those of the United States Government or the University of California, and shall not be used for advertising or product endorsement purposes.

This work was performed under the auspices of the U. S. Department of Energy by the University of California, Lawrence Livermore National Laboratory under Contract No. W-7405-Eng-48.

This report has been reproduced directly from the best available copy.

Available electronically at http://www.doc.gov/bridge

Available for a processing fee to U.S. Department of Energy

And its contractors in paper from

U.S. Department of Energy

Office of Scientific and Technical Information

P.O. Box 62

Oak Ridge, TN 37831-0062

Telephone: (865) 576-8401

Facsimile: (865) 576-5728

E-mail: reports@adonis.osti.gov

Available for the sale to the public from

U.S. Department of Commerce

National Technical Information Service

5285 Port Royal Road

Springfield, VA 22161

Telephone: (800) 553-6847

Facsimile: (703) 605-6900

E-mail: orders@ntis.fedworld.gov

Online ordering: http://www.ntis.gov/ordering.htm

OR

Lawrence Livermore National Laboratory

Technical Information Department's Digital Library

http://www.llnl.gov/tid/Library.html 


\section{CALIBRATION RESULTS OF THE EBIT MEDIUM-ENERGY FLAT-FIELD SPECTROMETER USING THE LBL ADVANCED LIGHT SOURCE.}

Jaan K. Lepson*, Peter Beiersdorfer ${ }^{+}$, Gregory V. Brown ${ }^{+}$, Hui Chen ${ }^{+}$, Eric M.

Gullikson", Marilyn B. Schneider ${ }^{+}$, Steven B. Utter ${ }^{+}$, and Keith L. Wong ${ }^{+}$.

* Space Sciences Laboratory, University of California, Berkeley

${ }^{+}$Lawrence Livermore National Laboratory

${ }^{\#}$ Lawrence Berkeley National Laboratory

ABSTRACT

The relative instrument response function of a flat-field grating extreme ultraviolet spectrometer was determined using the ALS synchrotron source in the wavelength region 40-200 $\AA$. Details of the calibration procedure and results are given in the report. 


\section{INTRODUCTION}

Accurate analysis of extreme ultraviolet data used in studies of such phenomena as supernova remnants, stellar coronae (Mewe et al. 1995), and EarthSun interactions (Warren et al. 1998) requires an accurate and comprehensive catalogue of emission lines in this spectral region. We are using Lawrence Livermore National Laboratory's electron beam ion trap EBIT-II to compile a comprehensive catalogue of wavelengths and intensities for astronomically relevant ions in the EUV region (Beiersdorfer et al. 1999b, Lepson et al. 2000). Accurate determination of relative intensities is a crucial component of this effort, necessitating a calibration of the detection response of our instrument. As part of this effort we used the Lawrence Berkeley Laboratory's Advanced Light Source to calibrate the detector efficiency of the medium-wavelength $1200 \mathrm{line} / \mathrm{mm}$ flatfield spectrometer used on EBIT-II (Beiersdorfer et al. 1999a, Utter et al. 1999).

\section{EXPERIMENTAL TECHNIQUE}

Data were taken at Lawrence Berkeley National Laboratory's Advanced Light Source beamline 6.3.2. The ALS synchroton produces light over a broad range. A monochromator in the beamline selects light at several harmonics, one of which is selected by a filter. The LLNL spectrometer was bolted to the end of the beamline. See diagram of beamline 6.3.2 in Appendix A. We used a 1,200 line/mm grazing incidence spectrometer developed by Harada and Kita (1980; Nakano et al. 1984) with a $3^{\circ}$ an angle of incidence. Readout was taken with a liquid nitrogen cooled CCD camera with a resolution of 1,024 x 1,024 pixels.

Data are acquired by stepping the ALS beamline by a set $\Delta \lambda$ over a given range of wavelengths. Data from a single run of this process are shown in Figure 1 .

Several steps are required to complete analysis of the raw data from ALS. First, the data are plotted for each run and the areas of the peaks are calculated using the area function of the program IGOR. Note that the data in Fig. 1 include both first-order (narrowly-spaced) and second-order (widely-spaced) peaks. We took particular care to exclude data where orders overlapped in order to avoid the possibility that higher energies would contribute via higher order to the first order lines we are interested in. Separate analyses were conducted on first and second orders; only the first order was utilized for the purpose of this report. 


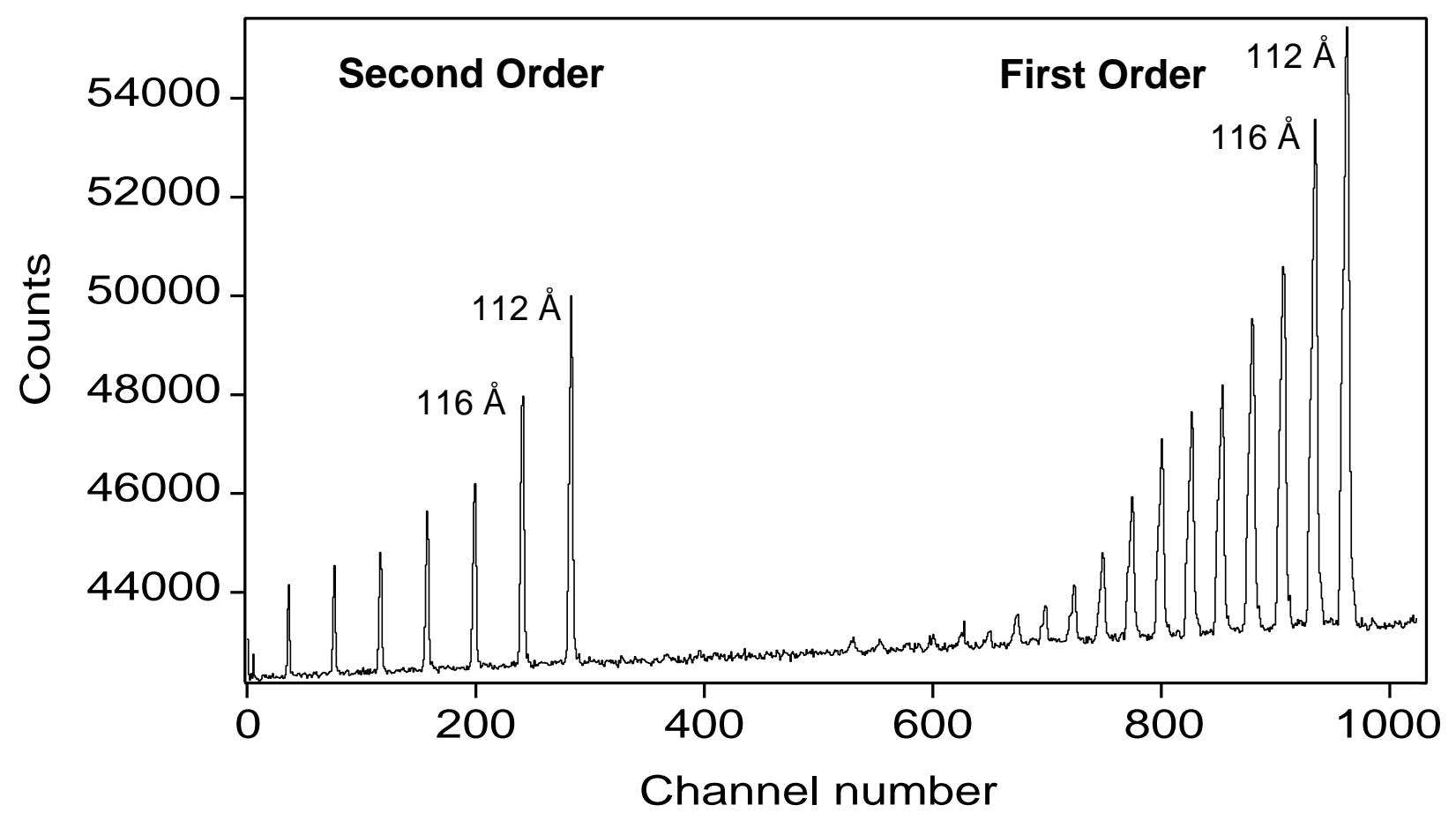

Figure 1. Plot of raw data from ALS using the 1200 line/mm flat-field grating. Peaks begin at $112 \AA$ with a separation of $4 \AA$.

The second step is to correct for the amount of beam intensity from the ALS. There are two detectors involved that monitor the ALS beamline emission. Detector 1 (Det.1) is upstream near the source, and is constantly monitoring the emission. Detector 2 (Det.2) is near the apparatus and monitors the amount of emission received by the spectrometer, but blocks the sightline and cannot be used when data are taken. Because of this, the correction must be done in two steps. Det.1 can be used while acquiring data, and the relative efficiency of Det.1 and Det. 2 can be calculated separately (Det.1/Det.2). The raw data are divided by Det.1 signal and then multiplied by the relative signal strength, Det.1/Det.2. This provides the equivalent of dividing by Det.2 signal. In other words, $($ Data/Det.1)*(Det.1/Det.2) $=$ Data/Det.2. Figure 2 shows the data set after being corrected for the amount of beam intensity from ALS. 


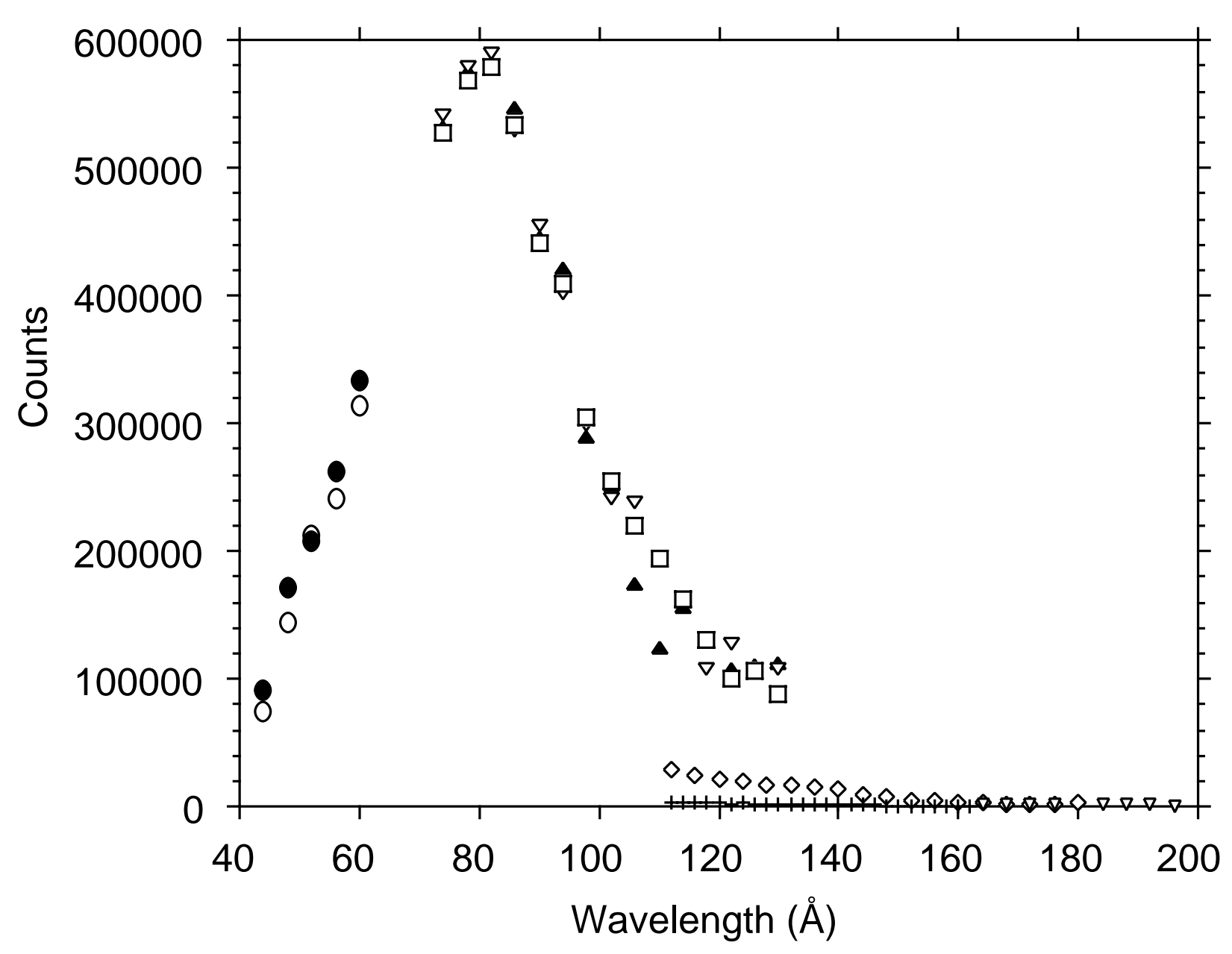

Figure 2. Plot of experimental runs after correction for amount of signal from ALS. Different symbols denote different runs utilized in this analysis.

Because some runs were exposed for longer periods than others, the third step is to correct for differing exposure times. This is done by simple multiplication so that the lines coincide. Figure 3 illustrates the data set after correction for exposure time. 


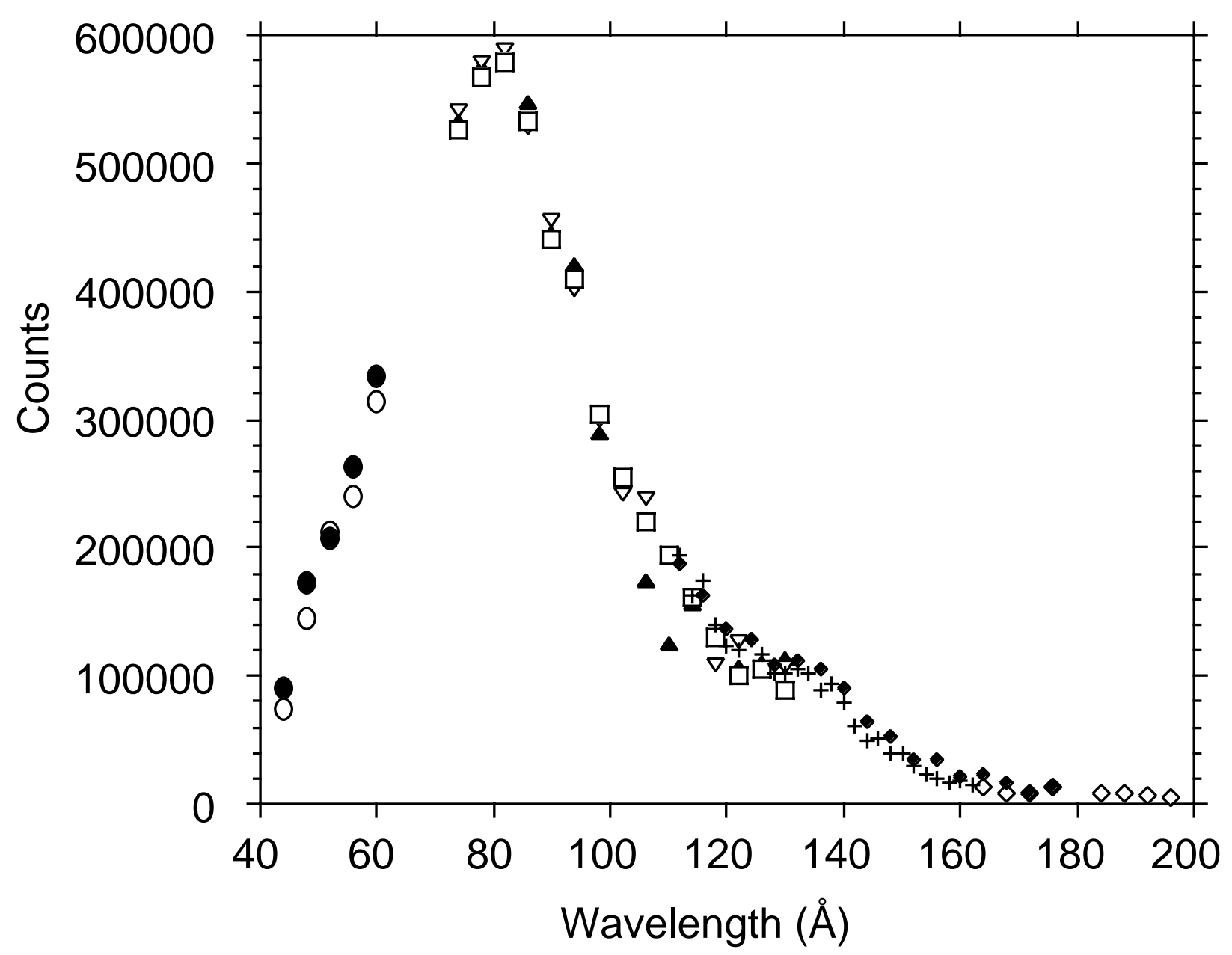

Figure 3. Full data set incorporating all runs used after manual correction for differing exposure times. Different symbols denote different runs utilized in this analysis.

After all this manipulation, the data still do not reflect the true response of the spectrometer. This is because the data are now normalized to the relative efficiency of Det.2. Detector 2 itself does not have a uniform response as a function of wavelength in this wavelength region. To get the actual spectrometer response, we need to adjust for the relative efficiency response of Det.2. This response is shown in Figure 4, as determined by Scholze et al. (2000). They did both a calculation and a measurement; we used their measurement. Our data ranged from $44 \AA$ to $200 \AA$, a region in which the responsivity function was relatively straightforward. We fit two separate curves to the responsivity function, one from $44 \AA$ to $100 \AA$, and one from $100 \AA$ to $200 \AA$. 




Figure 4. Responsivity function of the ALS diode in Detector 2.

Using the diode response, the detector-corrected data from Figure 3 are then divided by the diode responsivity function derived from Figure 4 to yield the actual spectrometer response. The peak of this data set is then normalized to 1, and is shown in Figure 5. A table of the response data is given in Appendix B. These data can be fit by two polynomial equations, one above $100 \AA$ and one from $100 \AA$ and below. For wavelengths $44 \AA \leq \lambda \leq 100 \AA$, the response is described by the equation $\mathrm{y}=-43.9928873+3.41086603 * \mathrm{x}-0.104141639 * \mathrm{x}^{2}+$ $0.0015626036 * x^{3}-0.0000114067305 * x^{4}+0.0000000322854769 * x^{5}$. For wavelengths $100 \AA<\lambda<200 \AA$, the response is described by the equation $\mathrm{y}=$ $25.4867357-0.783908447 * \mathrm{x}+0.00983263335^{*} \mathrm{x}^{2}-0.0000621519248 * \mathrm{x}^{3}+$ $0.000000196510498 * x^{4}-0.000000000247528158 * * x^{5}$. 




Figure 5. Normalized detector function of the LLNL EBIT $1200 / \mathrm{mm}$ flat field grating spectrometer.

\section{ACKNOWLEDGEMENTS}

This work was performed under the auspices of the Department of Energy by the University of California Lawrence Livermore National Laboratory. The work was supported in part by NASA High Energy Astrophysics and an IntraUniversity transfer agreement between LLNL and the Space Sciences Laboratory at the University of California, Berkeley. 


\section{REFERENCES}

Beiersdorfer, P., Crespo-López-Urritia, J. R., Springer, P., Utter., S. B., and Wong, K. L., 1999a, Rev. Sci. Instr. 70, 276.

Beiersdorfer, P., Lepson, J. K., Brown, G. V., Utter, S. B., Kahn, S. M., Liedahl, D. A., \& Mauche, C. W., 1999b, ApJ 519, L185.

Harada, T., and Kita, T., 1980, Appl. Opt. 19, 3987

Lepson, J.K., Beiersdorfer, P., Brown, G. V., Kahn, S. M., Liedahl, D. A., Mauche, C. W., \& Utter, S. B., 2000, RevMexAA 9, 137.

Mewe, R., Kaastra, J. S., Schrijver, C. J., van den Oord, G. H. J., \& Alkemade, F. J. M., 1995, A\&AS 296, 477.

Nakano, N., Kuroda, H., Kita, T., \& Harada, T., 1984, Appl. Opt. 232386.

Scholze, F., Henneken, H., Kuschnerus, P., Rabus, H., Richter, M., and Ulm, G., 2000, Nucl. Instr. \& Meth. A 439, 208.

Utter., S. B., Beiersdorfer, P., Brown, G. V., Clothiaux, E. J., .and Podder, N. K., 1999, Rev. Sci. Instr. 70, 284.

Warren, H. P., Mariska, J. T., \& Lean, J., 1998, J. Geophys. Res. 103, 12091 


\section{APPENDIX A}

Schematic diagram of the ALS Beamline 6.3.2 (www-cxro.lbl.gov/metrology)

50-1300 eV energy range

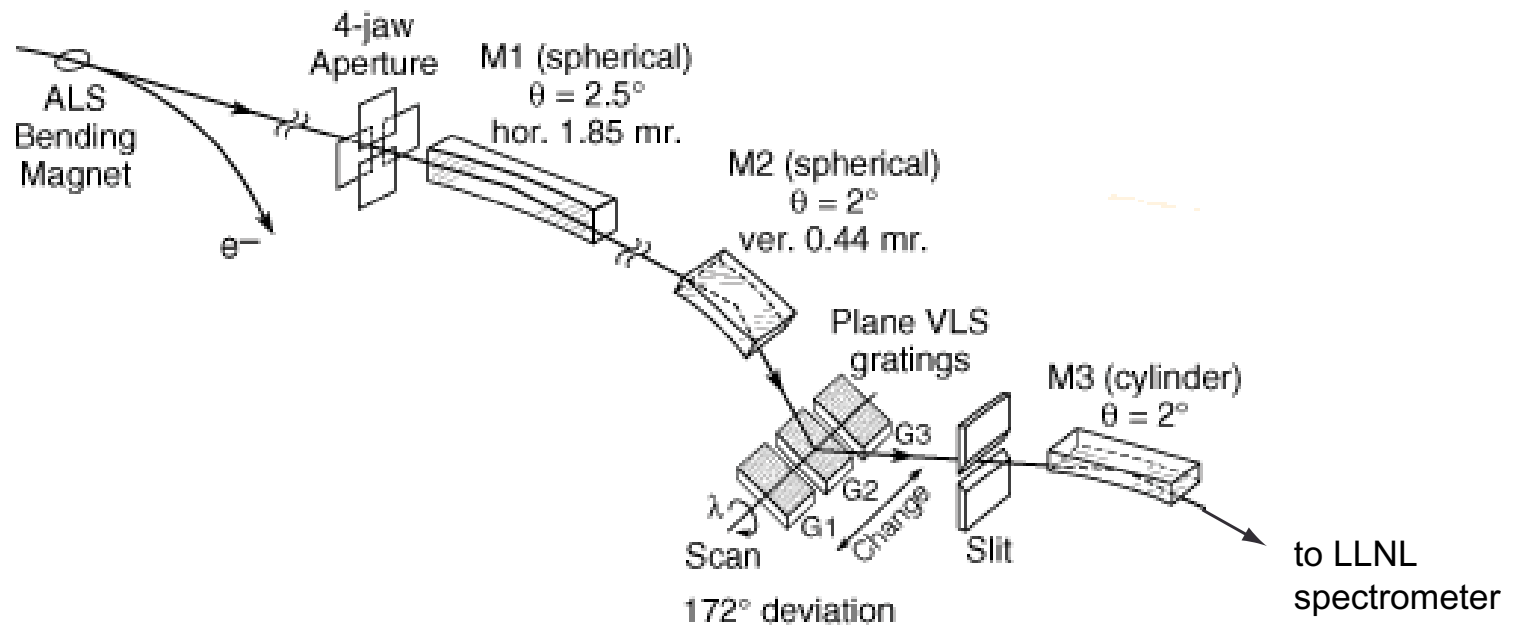




\section{APPENDIX B}

Responsivity of the EBIT medium-energy flat-field grating spectrometer by wavelength between 44 and $200 \AA$.

\begin{tabular}{|c|c|c|c|c|c|c|c|}
\hline $\begin{array}{r}\text { Wavelength } \\
(\AA)\end{array}$ & Responsivity & $\begin{array}{r}\text { Wavelength } \\
(\AA)\end{array}$ & Responsivity & $\begin{array}{r}\text { Wavelength } \\
(\AA)\end{array}$ & Responsivity & $\begin{array}{r}\text { Wavelength } \\
(\AA)\end{array}$ & Responsivity \\
\hline 44 & 0.147 & 81 & 0.998 & 121 & 0.176 & 161 & 0.026 \\
\hline 45 & 0.182 & 82 & 0.992 & 122 & 0.174 & 162 & 0.024 \\
\hline 46 & 0.215 & 83 & 0.983 & 123 & 0.173 & 163 & 0.022 \\
\hline 47 & 0.245 & 84 & 0.969 & 124 & 0.171 & 164 & 0.020 \\
\hline 48 & 0.272 & 85 & 0.952 & 125 & 0.168 & 165 & 0.019 \\
\hline 49 & 0.297 & 86 & 0.931 & 126 & 0.165 & 166 & 0.017 \\
\hline 50 & 0.321 & 87 & 0.906 & 127 & 0.162 & 167 & 0.016 \\
\hline 51 & 0.343 & 88 & 0.879 & 128 & 0.159 & 168 & 0.015 \\
\hline 52 & 0.364 & 89 & 0.849 & 129 & 0.155 & 169 & 0.014 \\
\hline 53 & 0.385 & 90 & 0.816 & 130 & 0.151 & 170 & 0.013 \\
\hline 54 & 0.406 & 91 & 0.782 & 131 & 0.147 & 171 & 0.012 \\
\hline 55 & 0.427 & 92 & 0.746 & 132 & 0.143 & 172 & 0.011 \\
\hline 56 & 0.448 & 93 & 0.709 & 133 & 0.138 & 173 & 0.011 \\
\hline 57 & 0.471 & 94 & 0.672 & 134 & 0.133 & 174 & 0.010 \\
\hline 58 & 0.494 & 95 & 0.636 & 135 & 0.127 & 175 & 0.010 \\
\hline 59 & 0.518 & 96 & 0.599 & 136 & 0.121 & 176 & 0.009 \\
\hline 60 & 0.543 & 97 & 0.564 & 137 & 0.116 & 177 & 0.009 \\
\hline 61 & 0.569 & 98 & 0.530 & 138 & 0.111 & 178 & 0.009 \\
\hline 62 & 0.597 & 99 & 0.498 & 139 & 0.106 & 179 & 0.009 \\
\hline 63 & 0.625 & 100 & 0.468 & 140 & 0.101 & 180 & 0.009 \\
\hline 64 & 0.654 & 101 & 0.440 & 141 & 0.096 & 181 & 0.009 \\
\hline 65 & 0.684 & 102 & 0.414 & 142 & 0.092 & 182 & 0.009 \\
\hline 66 & 0.714 & 103 & 0.392 & 143 & 0.087 & 183 & 0.009 \\
\hline 67 & 0.745 & 104 & 0.371 & 144 & 0.083 & 184 & 0.009 \\
\hline 68 & 0.775 & 105 & 0.353 & 145 & 0.078 & 185 & 0.009 \\
\hline 69 & 0.805 & 106 & 0.338 & 146 & 0.074 & 186 & 0.009 \\
\hline 70 & 0.834 & 107 & 0.324 & 147 & 0.070 & 187 & 0.009 \\
\hline 71 & 0.861 & 108 & 0.312 & 148 & 0.066 & 188 & 0.009 \\
\hline 72 & 0.887 & 109 & 0.301 & 149 & 0.062 & 189 & 0.008 \\
\hline 73 & 0.912 & 110 & 0.291 & 150 & 0.058 & 190 & 0.008 \\
\hline 74 & 0.933 & 111 & 0.281 & 151 & 0.055 & 191 & 0.008 \\
\hline 75 & 0.953 & 112 & 0.271 & 152 & 0.051 & 192 & 0.008 \\
\hline 76 & 0.969 & 113 & 0.261 & 153 & 0.048 & 193 & 0.007 \\
\hline 77 & 0.982 & 114 & 0.251 & 154 & 0.045 & 194 & 0.007 \\
\hline 78 & 0.992 & 115 & 0.239 & 155 & 0.042 & 195 & 0.006 \\
\hline 79 & 0.998 & 116 & 0.226 & 156 & 0.039 & 196 & 0.006 \\
\hline \multirow[t]{4}{*}{80} & 1.000 & 117 & 0.213 & 157 & 0.036 & 197 & 0.005 \\
\hline & & 118 & 0.200 & 158 & 0.033 & 198 & 0.004 \\
\hline & & 119 & 0.187 & 159 & 0.031 & 199 & 0.002 \\
\hline & & 120 & 0.176 & 160 & 0.028 & 200 & 0.001 \\
\hline
\end{tabular}

